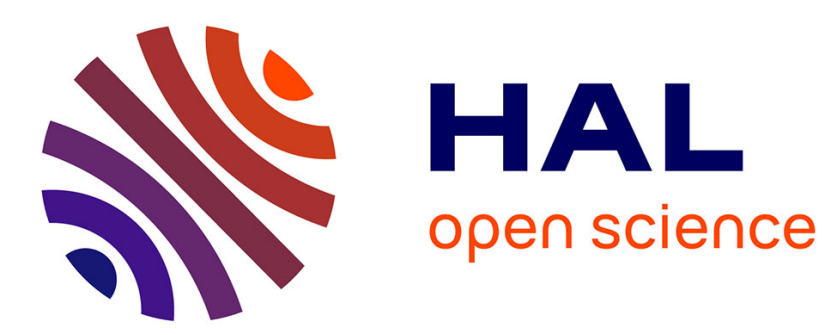

\title{
N Dimensional Crowd Motion
}

Jean-Paul Zolésio, Paola Goatin

\section{To cite this version:}

Jean-Paul Zolésio, Paola Goatin. N Dimensional Crowd Motion. 25th System Modeling and Optimization (CSMO), Sep 2011, Berlin, Germany. pp.557-566, 10.1007/978-3-642-36062-6_56 . hal01347584

\section{HAL Id: hal-01347584 \\ https://inria.hal.science/hal-01347584}

Submitted on 21 Jul 2016

HAL is a multi-disciplinary open access archive for the deposit and dissemination of scientific research documents, whether they are published or not. The documents may come from teaching and research institutions in France or abroad, or from public or private research centers
L'archive ouverte pluridisciplinaire HAL, est destinée au dépôt et à la diffusion de documents scientifiques de niveau recherche, publiés ou non, émanant des établissements d'enseignement et de recherche français ou étrangers, des laboratoires publics ou privés.

\section{(c)(1)}

Distributed under a Creative Commons Attribution| 4.0 International License 


\title{
$\mathrm{N}$ dimensional Crowd Motion
}

\author{
Jean-Paul Zolésio*and Paola Goatin ${ }^{\dagger}$
}

\begin{abstract}
We propose a variational formulation of a macroscopic model for crowd motion involving a conservation law describing mass conservation coupled with an eikonal equation giving the flow direction. To get a self contain paper we recall many results concerning flow mapping and convection process associated with non smooth vector field $V$.
\end{abstract}

\section{The crowd motion problem}

We consider a set $\Omega \subset \mathbb{R}^{N}$ representing a room and we denote by $\Gamma=\Gamma_{w} \cup \Gamma_{o}$ its boundary, where $\Gamma_{w}$ represents the solid wall, and $\Gamma_{o}$ the doors (we also assume $\left.\Gamma_{w} \cap \Gamma_{o}=\emptyset\right)$. The natural setting for the problem considered in this paper is $N=2$, but the 3D problem arises for example for fishes in an aquarium or flock of birds in a portion of sky.

The aim of this paper is to present a variational formulation of a model describing the motion of pedestrians in a finite set $\Omega$. The control parameter of the crowd dynamics is the speed vector field $V$ (which is time dependent) and equation (2.1) below expresses the conservation of the pedestrian mass $\int_{\Omega} \rho(t, x) d x$, where $\rho=\rho(t, x)$ denotes the pedestrian density. We study the dynamic system on a time interval $I=(0, \tau)$, the final time $\tau$ being arbitrary.

\section{Crowd motion}

We denote by $v \in \mathbb{R}^{N}$ the velocity, which is the norm of speed vector $V$, i.e. $v(t, x)=$ $|V(t, x)|$. The conservation of the mass, $\rho$ being the density, is classically expressed by the following equation in conservation form

$$
\begin{array}{ll}
\rho_{t}+\operatorname{div}(\rho V)=0, & \text { in } I \times \Omega, \\
\rho(0)=\rho_{0}, & \\
V . n=0, & \text { on } \Gamma_{w}, \\
V=v \vec{n}, \quad v \geq 0, & \text { on } \Gamma_{o} .
\end{array}
$$

${ }^{*}$ CNRS and INRIA, CNRS-INLN, 1136 route des Lucioles, 06902 Sophia Antipolis Cedex, France, Jean-Paul.Zolesio@inln.cnrs.fr

${ }^{\dagger}$ INRIA Sophia Antipolis - Méditerranée, 2004, route des Lucioles - BP 93, 06902 Sophia Antipolis Cedex, France, paola.goatin@inria.fr 
The "crowd rheology" modeling is done in two steps. First, we impose that $v$ is a decreasing function of the density $\rho$, which means that the pedestrian is going faster when there are less people around him. We assume given a decreasing function $f$ (we will consider as an example the function $f(\rho)=1-\rho$, so that we have

$$
v=f \circ \rho .
$$

Secondly, we want to take into account the fact that pedestrians try to minimize their travel time. As a consequence, they prefer avoiding high density regions, where they would proceed at low velocity. This behavior can be recovered by means of an eikonal equation whose running cost is given by the reciprocal of the velocity, as proposed by [3]. More precisely, we impose that there exists some potential function $\Phi$, which solves

$$
\begin{array}{ll}
\|\nabla \Phi\|=\frac{1}{f(\rho)}, & \text { in } \Omega, \\
\Phi=0, & \text { on } \Gamma_{o},
\end{array}
$$

such that

$$
V=f^{2}(\rho) \nabla \Phi
$$

Since the geometrical domain $\Omega$ is assumed to be simply connected, the existence of $\Phi$ such that (2.4) holds implies the following curl free condition (assuming that $f$ never reaches zero):

$$
\operatorname{curl}\left(\frac{V}{f^{2}(\rho)}\right)=0
$$

We observe that $\operatorname{curl}\left(\frac{V}{f^{2}(\rho)}\right)=f^{-2} \operatorname{curl} V+\nabla\left(f^{-2}\right) \times V$, then $(2.5)$ is equivalent to

$$
f(\rho) \operatorname{curl} V-2 f^{\prime}(\rho) \nabla \rho \times V=0,
$$

which, taking $f=1-\rho+\kappa$ (for some constant $\kappa>0$ ), simplifies to the following bilinear condition:

$$
(1-\rho) \operatorname{curl} V+2 \nabla \rho \times V=0 \text {. }
$$

Concerning the boundary condition for the vector field $V$, we shall assume that the initial density $\rho_{0}$ is compactly supported inside the domain $\Omega$ so that during the time $\tau$, the speed of the crowd being bounded, no pedestrian will reach the boundary so that without any loss of generality and for sake of simplicity we shall assume $V . n=0$ on the wall $\Gamma_{w}$ and $\|V\| \leq v_{\max }$, where $v_{\max }>0$ is the maximum speed for a pedestrian. The "strong" boundary bondition $\Phi=0$ on $\Gamma_{0}$ is lost in this process but is preserved in weak form as, from $V=v \vec{n}$ on $\Gamma_{0}$, we get $\Phi=c^{t e}$ on $\Gamma_{0}$.

\section{Speed vector $V$}

We assume $\Omega$ to be a bounded domain in $\mathbb{R}^{N}$ with "smooth boundary" $\Gamma$. We consider a vector field $V \in L^{1}\left(I, L^{1}\left(\Omega ; \mathbb{R}^{N}\right)\right)$ with divergence $\operatorname{div} V \in L^{1}\left(I, L^{1}(\Omega)\right)$ and normal 
component $V . n=0$ at the boundary (as an element of $W^{-1,1}(\Gamma)$ ). As a definition we set $E(V)$ the family of such $L^{1}$ vector fields $V$. We denote by $V(t)$ the partial mapping $x \rightarrow V(t)(x)=V(t, x)$.

\subsection{Regularization}

We assume now $\Omega$ to be star shaped, and without any loss of generality we assume that $0 \in \Omega$ and the domain to be star shaped with respect to 0 . (In fact in all what follows in this section it suffices the domain to be locally star shaped.) We denote by $V^{e}$ the extension of $V$ to $R^{N}$ by zero outside of $\Omega$. And we set

$$
\bar{V}_{n}(t, x)=V^{e}(t,(1+1 / n) x),
$$

which is compactly supported in $\Omega$. Let $\lambda_{n}$ be a mollifier and consider

$$
\left.V_{n}(t)=\lambda_{n} \star \bar{V}_{n}(t) \in C_{c}^{\infty}\left(\Omega, \mathbb{R}^{N}\right)\right)
$$

We assume the mollifier suitably chosen, so that $V_{n}(t)$ is also compactly supported in $\Omega$. We get $\operatorname{div} \bar{V}_{n}(t, x)=(1+1 / n)(\operatorname{div} V)^{e}(t,(1+1 / n) x)$

$$
\operatorname{div} V_{n}(t)=(1+1 / n) \lambda_{n} \star \operatorname{div} \bar{V}_{n}
$$

So that we have the following strong convergences

$$
V_{n} \rightarrow V \text { in } L^{1}\left(I, L^{1}\left(\Omega, \mathbb{R}^{N}\right)\right) ; \quad \operatorname{div} V_{n} \rightarrow \operatorname{div} V \text { in } L^{1}\left(I, L^{1}(\Omega)\right)
$$

\section{Flow mapping}

Consider $V \in L^{1}\left(I, C^{1}(\Omega) \cap H_{0}^{1}(\Omega)\right)$. We prove here, following [6], that the mapping mapping $T_{t}(V)$ defined over the bounded domain $\Omega$.

\subsection{Existence}

Let $X \in \Omega$, we consider the sequence

$$
\begin{aligned}
x_{0}(t) & =X \\
x_{1}(t) & =X+\int_{0}^{t} V\left(s, x_{0}(s)\right) d s \\
& \vdots \\
x_{n+1}(t) & =X+\int_{0}^{t} V\left(s, x_{n}(s)\right) d s
\end{aligned}
$$

As a first result we have $x_{n}(t) \in \bar{\Omega}$. We apply Ascoli compactness theorem to the family $x_{n}(.) \in C^{0}(I, \bar{\Omega})$. We verify the equicontinuity of this family at any $t \in I=[0, \tau]$ :

$$
x_{n}(t+\varepsilon)-x_{n}(t)=\int_{t}^{t+\varepsilon} V\left(s, x_{n}(s)\right) d s
$$




$$
\forall n, \quad\left\|x_{n}(t+\varepsilon)-x_{n}(t)\right\| \leq \int_{t}^{t+\varepsilon}\|V(s)\|_{L^{\infty}\left(\Omega, \mathbb{R}^{N}\right)} d s
$$

Then this sequence converges in $C^{0}(I, \bar{\Omega})$ to an element $x(t)$, passing to the limit in (4.1) we observe that $x(t)$ is a solution to the flow equation.

\subsection{Uniqueness}

Assume $x^{i}, i=1,2$ are two solutions. We set $y(t)=x^{2}(t)-x^{1}(t)$, we get

$$
y(t)=\int_{0}^{t}\left[\int_{0}^{1} D V\left(s, x^{1}(s)+\lambda y(s)\right) d \lambda\right] \cdot y(s) d s
$$

Then

$$
\forall t \in I, \quad\|y(t)\| \leq \int_{0}^{t}\|D V(s)\|_{L^{\infty}}\|y(s)\| d s \leq \max _{s}\|y(s)\| \int_{0}^{t}\|D V(s)\|_{L^{\infty}} d s
$$

Choose $t_{V}$ such that $k=\int_{0}^{t_{V}}\|D V(s)\|_{L^{\infty}} d s<1$, then we get $y=0$ on $\left[0, t_{V}\right]$. Then the solution is unique on this interval $\left[0, t_{V}\right]$. Now the interval $I$ can be decomposed in a finite number of such intervals, then the solution is unique on $I$.

Let $X \in \Omega$, we set $T_{t}(V)(X)=x(t)$, and $T(V)$ denotes the mapping $(t, X) \mapsto$ $T_{t}(V)(X)$

Proposition 4.1. Let $V \in L^{1}\left(I, C^{1}(\Omega) \cap H_{0}^{1}(\Omega)\right)$, the flow mapping $T_{t}(V)$ is defined for any $t \leq \tau$ and $T_{t}(V) \in C^{1}(\Omega)$. It is invertible and $T_{t}(V)^{-1}=T_{t}\left(V^{t}\right)$ where $V^{t}(s)=-V(t-s)$, so that $T_{t}(V)^{-1} \in C^{1}(\Omega)$.

\subsection{Convection}

Let $\zeta_{0} \in L^{1}(\Omega)$ and set $\zeta(t)=\zeta_{0} \circ T_{t}(V)^{-1}$. This function solves the convection problem

$$
\zeta_{t}+\nabla \zeta(t) \cdot V(t)=0, \zeta(0)=\zeta_{0}
$$

Moreover, as $\zeta_{t}=-\operatorname{div}(\zeta V)+\zeta \operatorname{div} V(t) \in L^{1}\left(I, W^{-1,1}(\Omega)\right)$, we get $\zeta \in C\left(I, W^{-1,1}(\Omega)\right)$.

\section{$5 \quad$ Solution to transport equation (2.1)}

\subsection{The homogeneous equation}

Proposition 5.1. Let $V \in L^{1}\left(I, L^{1}\left(\Omega, \mathbb{R}^{N}\right)\right)$ with div $V \in L^{1}(I \times \Omega)$ and V.n $=0$ on $\Gamma$ as an element of $W^{-1,1}(\Gamma)$. Let $\rho_{0} \in L^{\infty}(\Omega)$. Then there exists a solution $\rho \in L^{\infty}(I \times \Omega)$ to the transport equation (4.2). Moreover, we have the following estimates

$$
\|\rho\|_{L^{\infty}(I \times D)} \leq\left\|\rho_{0}\right\|_{L^{\infty}(\Omega)}
$$


Proof. Let $V_{n}$ a smooth field strongly converging to $V$. Let $\rho_{n}$ be the solution of this equation associated to the smooth vector field $V_{n}$, that is:

$$
\rho_{n}(t)=\rho_{0} \circ T_{t}\left(V_{n}\right)^{-1}
$$

then

$$
\left\|\rho_{n}\right\|_{L^{\infty}(I \times D)}=\left\|\rho_{0}\right\|_{L^{\infty}(\Omega)}
$$

There exists a subsequence which is $\sigma *$ weakly converging to some element $\rho$ verifying $\|\rho\|_{L^{\infty}(I \times D)} \leq\left\|\rho_{0}\right\|_{L^{\infty}(\Omega)}$ and we can pass to the limit in the weak formulation :

$$
\forall \varphi \in C^{\infty}(I \times \Omega), \varphi(\tau)=0, \quad \int_{0}^{\tau} \int_{\Omega} \rho_{n}\left(-\varphi_{t}-\operatorname{div}\left(\varphi V_{n}\right)\right) d x d t+\int_{\Omega} \rho_{0} \varphi(0) d x=0
$$

\subsection{The non-homogeneous equation}

Proposition 5.2. Let $V \in L^{1}\left(I, L^{1}\left(\Omega, \mathbb{R}^{N}\right)\right)$ with div $V \in L^{1}(I \times \Omega)$ and $V . n=0$ on $\Gamma$ as an element of $W^{-1,1}(\Gamma)$. Let $\rho_{0} \in L^{\infty}(\Omega)$, $\left\|\rho_{0}\right\| \leq 1$. Assume that $F \in$ $L^{1}\left(I, L^{\infty}(\Omega)\right)$, then there exists a solution $\rho \in L^{\infty}(I \times \Omega)$ to the transport equation

$$
\rho_{t}+\nabla \rho . V=F, \quad \rho(0)=\rho_{0}
$$

Moreover, we have the following estimate

$$
\|\rho\|_{L^{\infty}(I \times D)} \leq\left\|\rho_{0}\right\|_{L^{\infty}(\Omega)}+\int_{0}^{\tau}\|F(s)\|_{L^{\infty}(\Omega)} d s .
$$

Proof. Let $V_{n}$ be a smooth field strongly converging to $V$. Let $\rho_{n}$ be the solution associated to the smooth vector field $V_{n}$, that is:

$$
\rho_{n}(t)=\left[\rho_{0}+\int_{0}^{t} F(s) \circ T_{s}\left(V_{n}\right) d s\right] \circ T_{t}\left(V_{n}\right)^{-1}
$$

then

$$
\begin{aligned}
\forall t, \quad\left\|\rho_{n}(t)\right\|_{L^{\infty}(\Omega)} & =\left\|\rho_{0}+\int_{0}^{t} F(s) \circ T_{s}\left(V_{n}\right) d s\right\|_{\left.L^{\infty} \Omega\right)} \\
& \leq\left\|\rho_{0}\right\|_{L^{\infty}(\Omega)}+\int_{0}^{t}\|F(s)\|_{L^{\infty}(\Omega)} d s .
\end{aligned}
$$

Then

$$
\left\|\rho_{n}(t)\right\|_{L^{\infty}(I \times \Omega)} \leq\left\|\rho_{0}\right\|_{L^{\infty}(\Omega)}+\int_{0}^{\tau}\|F(s)\|_{L^{\infty}(\Omega)} d s
$$

The weak formulation gives

$$
\varphi(\tau)=0, \quad \int_{0}^{\tau} \int_{\Omega} \rho_{n}\left(-\varphi_{t}-\operatorname{div}\left(\varphi V_{n}\right)\right) d x d t+\int_{\Omega} \rho_{0} \varphi(0, x) d x=\int_{0}^{\tau} \int_{\Omega} F \varphi d x d t
$$


for all $\varphi \in C^{\infty}(I \times \Omega)$. Now

$$
\operatorname{div}\left(\varphi V_{n}\right)=\varphi \operatorname{div} V_{n}+\nabla \varphi \cdot V_{n} \longrightarrow \operatorname{div}(\varphi V) \quad \text { in } L^{2},
$$

which, together with the weak convergence of $\rho_{n}$ to some $\rho$, enables us to pass to the limit and obtain the weak formulation of a solution $\rho$ to equation (5.5). The bound leads to the convergence (up to a subsequence) weakly in $\sigma-*$, and the (weak) limit preserves the estimate.

\section{The conservation equation}

Proposition 6.1. Let $\rho_{0} \in L^{\infty}(\Omega)$ and $V \in L^{1}\left(I \times \Omega, \mathbb{R}^{N}\right)$, div $V \in L^{1}\left(I, L^{\infty}(\Omega)\right)$, with V.n $=0$ in $W^{-1,1}(\Gamma)$. Assuming $\|\operatorname{div} V\|_{L^{1}\left(I, L^{\infty}(\Omega)\right)}<1$, there exists a solution $\rho \in L^{\infty}(I \times \Omega)$ to equation $(2.1)$.

Proof. Equation (2.1) writes

$$
\rho_{t}+\nabla \rho . V=-\rho \operatorname{div} V, \quad \rho(0)=\rho_{0} .
$$

Let

$$
\rho_{t}^{n+1}+\nabla \rho^{n+1} \cdot V=-\rho^{n} \operatorname{div} V, \quad \rho^{n+1}(0)=\rho_{0}
$$

and

$$
\delta_{n, p}=\rho^{n+p}-\rho^{n} .
$$

From ??, as $\delta_{n, p}(0)=0$, we get,

$$
\begin{aligned}
\left\|\delta_{n+1, p}\right\|_{L^{\infty}\left(I, L^{\infty}\right)} & \leq\left\|\delta_{n, p} \operatorname{div} V\right\|_{L^{1}\left(I, L^{\infty}\right)} \\
& \leq\left\|\delta_{n, p}\right\|_{L^{\infty}\left(I, L^{\infty}\right)}\|\operatorname{div} V\|_{L^{1}\left(I, L^{\infty}(\Omega)\right)} \\
& \leq\left\|\delta_{n-1, p}\right\|_{L^{1}\left(I, L^{\infty}\right)}\|\operatorname{div} V\|_{L^{1}\left(I, L^{\infty}(\Omega)\right.}^{2} \\
& \cdots\left\|\delta_{1, p}\right\|_{L^{\infty}\left(I, L^{\infty}(\Omega)\right)}\|\operatorname{div} V\|_{L^{1}\left(I, L^{\infty}(\Omega)\right)}^{n} \\
& =\left\|\rho^{p+1}-\rho^{1}\right\|_{L^{\infty}\left(I, L^{\infty}(\Omega)\right)}\|\operatorname{div} V\|_{L^{1}\left(I, L^{\infty}(\Omega)\right)}^{n}
\end{aligned}
$$

Now

$$
\begin{aligned}
& \left\|\rho^{p+1}\right\|_{L^{\infty}\left(I, L^{\infty}(\Omega)\right)} \\
& \leq\left\|\rho_{0}\right\|_{L^{\infty}(\Omega)}+\left\|\rho^{p} \operatorname{div} V\right\|_{L^{1}\left(I, L^{\infty}(\Omega)\right)} \\
& \leq\left\|\rho_{0}\right\|_{L^{\infty}(\Omega)}+\left\|\rho^{p}\right\|_{L^{\infty}\left(I, L^{\infty}(\Omega)\right)}\|\operatorname{div} V\|_{L^{1}\left(I, L^{\infty}(\Omega)\right)} \\
& \leq\left\|\rho_{0}\right\|_{L^{\infty}(\Omega)}+\left(\left\|\rho_{0}\right\|_{L^{\infty}(\Omega)}+\left\|\rho^{p-1} \operatorname{div} V\right\|_{L^{1}\left(I, L^{\infty}(\Omega)\right)}\|\operatorname{div} V\|_{\mathrm{L}^{1}\left(I, L^{\infty}(\Omega)\right.}\right. \\
& \leq\left\|\rho_{0}\right\|_{L^{\infty}(\Omega)}+\left(\left\|\rho_{0}\right\|_{L^{\infty}(\Omega)}+\left\|\rho^{p-1}\right\|_{L^{1}\left(I, L^{\infty}(\Omega)\right)}\|\operatorname{div} V\|_{L^{1}\left(I, L^{\infty}(\Omega)\right)}\|\operatorname{div} V\|_{L^{1}\left(I, L^{\infty}(\Omega)\right)}\right. \\
& \cdots \\
& \quad \leq\left\|\rho_{0}\right\|_{L^{\infty}(\Omega)} \Sigma_{i=0, \ldots ., p+1}\|\operatorname{div} V\|_{L^{1}\left(I, L^{\infty}(\Omega)\right)}^{i}
\end{aligned}
$$

We get

$$
\forall p,\left\|\rho^{p}\right\|_{L^{\infty}\left(I, L^{\infty}(\Omega)\right)} \leq\left\|\rho_{0}\right\|_{L^{\infty}(\Omega)}\left(1-\|\operatorname{div} V\|_{L^{1}\left(I, L^{\infty}(\Omega)\right)}\right)^{-1} .
$$

So $\left\{\rho^{n}\right\}_{n}$ is a Cauchy sequence in $L^{\infty}\left(I, L^{\infty}(\Omega)\right)$. 
Theorem 6.1. Let $\rho_{0} \in L^{\infty}(\Omega)$ and $V \in L^{1}\left(I \times \Omega, \mathbb{R}^{N}\right)$, div $V \in L^{1}\left(I, L^{\infty}(\Omega)\right)$ with $V . n=0$ in $W^{-1,1}(\Gamma)$. Then there exists a solution $\rho \in L^{\infty}(I \times \Omega)$ to equation (2.1).

Proof. We consider a finite covering of the interval $I=[0, \tau]$ by open intervals $] t_{i}, t_{i}+$ $\tau_{i}\left[, i=0, \ldots, k\right.$, with $t_{0}=0, t_{k}+\tau_{k}=\tau$, and such that $\int_{t_{i}}^{t_{i}+\tau_{i}}\|V(t)\|_{L^{\infty}(\Omega)} d t<1$ for all $i$. From the next proposition, there exists a solution $\rho_{1}$ on the interval $] t_{0}, t_{0}+\tau_{0}[$ verifying $\rho_{1}(0)=\rho_{0}$. This solution is continuous in the following sense:

$$
\rho_{1} \in C\left(\left[t_{0}, t_{0}+\tau_{0}\right], W^{-1,1}(\Omega)\right) .
$$

Then for all $t \in\left[t_{0}, t_{0}+\tau_{0}\right]$ the element $\rho_{1}(t)$ is defined as an element of $W^{-1,1}(\Omega)$, but for a.e. $t \in\left[t_{0}, t_{0}+\tau_{0}\right]$ this element is in $L^{\infty}(\Omega)$. So we can choose such an element $\left.\tilde{t}_{1} \in\right] t_{1}, t_{0}+\tau_{0}\left[\right.$ with $\rho_{1}\left(\tilde{t}_{1}\right) \in L^{\infty}(\Omega)$. Then on the interval $I_{2}=\left(\tilde{t}_{1}, t_{1}+\tau_{1}\right)$ by the next proposition we built a solution $\rho_{2}$, and so on on each interval $I_{i}$. We obtain a solution on the whole interval $(0, \tau)$.

\section{Crowd motion variational formulation}

Let us denote by $I$ the time interval, $I=] 0, \tau[$. To any element $V \in E(\Omega) \subset$ $L^{1}\left(I, L^{1}\left(\Omega, R^{N}\right)\right)$, we associate the set $R_{V}$ of solutions to the conservation equation (2.1) and we introduce the functionals

$$
\begin{gathered}
J(V, \rho)=\|f \circ \rho-|V|\|_{L^{1}\left(I, L^{1}(\Omega)\right)}+\beta\|(1-\rho) \operatorname{curl} V+2 \nabla \rho \times V\|_{L^{1}\left(I, W^{-1,1}\left(\Omega, \mathbb{R}^{N}\right)\right)} \\
j(V)=\inf _{\rho \in R_{V}} J(V, \rho)
\end{gathered}
$$

which can be rewritten as

$$
j(V)=\inf _{\left.r \in L^{\infty}\left(I, L^{\infty} \Omega\right)\right)} \sup _{\theta \in \mathcal{W}} L(V, r, \theta)
$$

where

$$
\mathcal{W}=\left\{\theta \in L^{1}\left(I, W^{1, \infty}(\Omega)\right) \cap W^{1,1}\left(I, L^{1}(\Omega)\right),: \theta(\tau)=0\right\}
$$

and

$$
\left.L(V, r, \theta)=J(V, r)+\int_{I} \int_{\Omega} r\left(\theta_{t}+\nabla \theta \cdot V\right)\right) d x d t+\int_{D} \rho_{0} \theta(0) d x .
$$

We have the obvious

Proposition 7.1. Let $V \in E(\Omega)$ such that $j(V)=0$, then it solves the crowd problem in the sense that there exists a solution $\rho \in R_{V}$ such that $(V, \rho)$ solves the crowd system (2.1), (2.3), (2.4).

If such a speed vector $V$ exists it minimizes the positive functional $j$ over the space $E(\Omega)$. The variational approach for the crowd problem under consideration is to replace it by the weaker one which is the minimization of the non negative functional $j$ over $E(\Omega)$. Our approach is now to compute the gradient of the functional $j$ to be minimized.

We remark that, if the infimum of the functional $j$ is not zero, then, in some sense, the crowd problem formulated as (2.1), (2.3), (2.4) would have no solution. If the infimum reaches zero it would built a solution. 


\section{Minimization of the functional}

The main objective is to calculate the gradient of $j$.

We denote by $j^{\prime}(V ; W)=\liminf _{\varepsilon>0, \varepsilon \rightarrow 0} j(V+\varepsilon W)$. If the limit exists, it is the classical Gateau semi derivative. For sake of simplicity let us first compute the gradient for a regularized functional $j_{\gamma}$ expressed in the following form

$$
j_{\gamma}(V)=j_{2}(V)+\frac{\gamma}{2} \int_{I} \int_{D}\|V(t)\|_{\mathcal{H}}^{2} d t
$$

where $\mathcal{H}$ stands here for a Banach space of function over the domain $\Omega$ which will ensure the set $R_{V}$ of solutions to the conservation equation (2.1) to be a singleton element $\rho_{V}$. This will be the case for the following choices :

$$
\mathcal{H}=\left\{V \in H^{3}\left(\Omega, R^{N}\right), \Delta v=0, V . n=v_{\max } \text { on } \Sigma\right\} \subset E(\Omega) \cap C^{1}(\bar{\Omega})
$$

or

$\mathcal{H}=\left\{V \in B V\left(\Omega, R^{N}\right)\right.$ with $\left.\operatorname{div} V \in L^{\infty}(\Omega)\right\}$ and where $j_{2}$ is the quadratic version of $j$, that is $j_{2}(V)=\inf _{\rho \in R_{V}} J_{2}(V, \rho)$ with

$$
J_{2}(V, \rho)=\|f \circ \rho-|V|\|_{L^{2}\left(I, L^{2}(\Omega)\right)}^{2}+\beta\|(1-\rho) \operatorname{curl} V+2 \nabla \rho \times V\|_{L^{2}\left(I, L^{2}\left(\Omega, \mathbb{R}^{N}\right)\right)}^{2}
$$

We briefly recall now the calculus of the gradient of the functional expressed in Min Max.

\subsection{Derivative of a function in Min Max form, from [1], [6]}

Let $E, F$ be two Banach spaces and $L(s, e, f)$ be a function defined from $[0,1] \times K_{E} \times K_{F}$ into $\mathbb{R}$, where $K_{E}, K_{F}$ are convex sets, respectively in $E$ and $F$. Assume that the Lagrangian functional $L$ is convex l.s.c. with repect to $e$, concave u.s.c. with respect to $f$ and continuously differentiable with respect to the parameter $s$. Assume moreover that there exists a non empty set $S(s)$ of saddle points. Then it always takes the following form:

$$
\begin{gathered}
S(s)=A(s) \times B(s), A(s) \subset K_{E}, B(s) \subset K_{F}, \text { such that }: \\
\forall a(s) \in A(s), \forall b(s) \in B(s), \forall \gamma \in K_{A}, \forall \beta \in K_{B}, \\
L(s, a(s), \beta) \leq L(s, a(s), b(s)) \leq L(t, \gamma, b(s))
\end{gathered}
$$

So that $\forall \gamma^{\prime} \in K_{E}, \forall \beta^{\prime} \in K_{F}$ we have

$$
-L\left(0, \gamma^{\prime}, b(0)\right) \leq-L(0, a(0), b(0)) \leq-L\left(0, a(0), \beta^{\prime}\right)
$$

By choosing $\gamma=a(0), \beta=b(0), \gamma^{\prime}=a(s), \beta^{\prime}=b(s)$, and adding the two previous inequalities we get for any $s>0$ :

$$
\begin{aligned}
\frac{L(s, a(s), b(0))-L(0, a(s), b(0))}{s} & \leq \frac{L(s, a(s), b(s))-L(0, a(0), b(0))}{s} \\
& \leq \frac{L(s, a(0), b(s))-L(0, a(0), b(s))}{s}
\end{aligned}
$$


Under reasonable smoothness assumptions on $L$ and Kuratowski continuity of the sets $A(s)$ and $B(s)$ we get the semi-derivative of

$$
l(s)=\min _{a \in K_{E}} \max _{b \in K_{F}} L(s, a, b)
$$

i.e.

$$
l^{\prime}(0)=\min _{a \in A(0)} \max _{b \in B(0)} \frac{\partial}{\partial s} L(0, a, b)
$$

In the following section we shall make use of that semi-derivative in the specific situation in which the set $S(0)$ is reduced to a unique pair, $A(0)=\{y\}, B(0)=\{p\}$, where $y$ and $p$ will be the "state" and "adjoint-state" solution associated with the wave equation under consideration. In this situation the function $l$ is differentiable at $s=0$ and the derivative (8.3) takes the following form:

$$
l^{\prime}(0)=\frac{\partial}{\partial s} L(0, y, p)
$$

\subsection{The optimal system}

Just for shortness of the expressions we make $\beta=0$ and $m=1$ so that

$J_{\gamma}^{\prime}(V ; W)=\int_{I} \int_{\Omega}\left(2\left(|V|-f \circ \rho_{V}\right) \frac{V}{|V|} \cdot W+\rho_{V} W . \nabla P\right) d x d t+\gamma \int_{I} \int_{\Omega} D \Delta V . D \Delta W d x d t$,

where the adjoint state $P$ is the solution to the following backward adjoint problem:

$$
P_{t}+\nabla P . V=2\left(f \circ \rho_{V}-|V|\right) f^{\prime} \circ \rho_{V}, \quad P(\tau)=0 .
$$

Obviously the gradient is

$$
\nabla J_{\epsilon}(V)=2\left(|V|-f \circ \rho_{V}\right) \frac{V}{|V|}+\rho_{V} \nabla P-\gamma \Delta V .
$$

Proposition 8.1. If the vector field $V$ minimizes the functional $j_{\gamma}$, then it solves the optimality system

$$
\begin{array}{cc}
\rho_{t}+\operatorname{div}(\rho V)=0, & \rho(0)=\rho_{0}, \\
& P_{t}+\nabla P . V=-2(1-\rho+\gamma-|V|), \quad P(\tau)=0, \\
& \left(2(|V|-f \circ \rho) \frac{V}{|V|}+\rho \nabla P-\gamma \Delta V=0 .\right. \\
V(t) . n=0 \text { on } \Gamma_{\omega}, V(t) \cdot n=v_{\text {max }} \text { on } \Gamma_{0}, \quad \Delta V(t)=\Delta^{2} V(t)=0, \text { on } \Sigma
\end{array}
$$




\section{References}

[1] M. Cuer and J.P. Zolesio Control of singular problem via differentiation of a min-max, Systems \& Control Letters, 11(2), 1988, 151-158.

[2] M.C. Delfour and J.P. Zolésio, Shapes and Geometries: Analysis, Differential Calculus and Optimization, SIAM series on Advances in Design and Control, Society for Industrial and Applied Mathematics, Philadelphia, USA (2001) second ed. 2011.

[3] R.L. Hughes, A continuum theory for the flow of pedestrians, Transpn. Res.,B, $36(6), 2002,507-535$.

[4] J. Sokolowski and J.P. Zolésio, Introduction to shape optimization. Shape sensitivity analysis, Springer Ser. Comput. Math., 16. Springer-Verlag, Berlin, 1992.

[5] J.P. Zolésio, The material derivative (or speed) method for shape optimization, in "Optimization of distributed parameter structures", Vol. II (Iowa City, Iowa, 1980), E. J. Haug and J. Céa, eds., pp. 1089-1151, NATO Adv. Sci. Inst. Ser. E: Appl. Sci., 50, Sijhofff and Nordhoff, Alphen aan den Rijn, 1981 (Nijhoff, The Hague).

[6] Control of moving domains, shape staibilzation and variational tube formulation, Int. series of numerical mathematics, vol 55, p. 329-382, 2007, Birkhause Verlag Basel/Switzerland 